\title{
Some Subtleties in the Relationships among Heat Kernel Invariants, Eigenvalue Distributions, and Quantum Vacuum Energy version of 28 November 2014
}

\author{
S A Fulling ${ }^{1}$ and Yunyun Yang ${ }^{2}$ \\ ${ }^{1}$ Departments of Mathematics and Physics, Texas A\&M University, College Station, \\ TX 77843-3368 USA \\ ${ }^{2}$ Department of Mathematics, Louisiana State University, Baton Rouge, LA 70803 \\ USA \\ E-mail: fulling@math.tamu.edu, yyang18@math.lsu.edu
}

\begin{abstract}
A common tool in Casimir physics (and many other areas) is the asymptotic (high-frequency) expansion of eigenvalue densities, employed as either input or output of calculations of the asymptotic behavior of various Green functions. Here we clarify some fine points and potentially confusing aspects of the subject. In particular, we show how recent observations of Kolomeisky et al. [Phys. Rev. A 87 (2013) 042519] fit into the established framework of the distributional asymptotics of spectral functions.

PACS numbers: 02.30.Lt, 03.65.Sq, 11.10.Gh
\end{abstract}

AMS classification scheme numbers: 34B27, 35P20, 46F10, 81T55

Submitted to: J. Phys. A: Math. Gen. 


\section{Introduction}

The density of eigenvalues of a differential operator (such as a Hamiltonian), with the closely related subjects of semiclassical approximations and the asymptotic dependence of various Green functions on various coordinates and parameters, is a central tool in nuclear, atomic, and molecular physics [1], condensed-matter physics [2], generalrelativistic quantum field theory [3], and quantum vacuum energy (Casimir physics) [4]. (These four references are merely illustrative.) A key point is that a variety of differential equations (relativistic and nonrelativistic, classical and quantum) are all associated with the same elliptic, spatial differential operator; a variety of Green functions (heat, Schrödinger, wave, ...) are related to the same eigenvalue distribution and hence to each other, and the study of one may yield valuable insight into another. Nevertheless, the subject contains some complications and pitfalls that are likely occasionally to rise up and confuse even those of us who think we have become experts in it.

Here is a synopsis of the paper in mathematical language: Let $-H$ be the Laplacian on scalar functions in a compact region in $\mathbf{R}^{\mathbf{3}}$ with smooth Dirichlet boundary; let $K(t)=\operatorname{Tr} e^{-t H}$ be the heat kernel trace, $T(t)=\operatorname{Tr} e^{-t \sqrt{H}}$ the trace of the cylinder (Poisson) kernel, and $N\left(\omega^{2}\right)$ the eigenvalue counting function. Loosely speaking, the small- $t$ asymptotics of $K$ and $T$ are in close correspondence with the averaged large- $\omega$ asymptotics of $N$, but there are some subtleties that can be confusing. (1) Nonnegative integer powers of $t$ in the expansion of $K$ do not correspond to (negative) integer powers of $\omega^{2}$ in the expansion of $d N / d\left(\omega^{2}\right)$ (even after the latter has been well defined by averaging). Instead, they give rise to terms $\delta^{(n)}\left(\omega^{2}\right)$ in the moment asymptotic expansion of a distribution (which is actually an expansion in a parameter, not in $\omega$ ). (2) The expansion of $T$ contains additional, nonlocal spectral invariants, which show up in $d N / d \omega=2 \omega d N / d\left(\omega^{2}\right)$, filling in the missing odd negative integer powers. The first of these, $O\left(t^{0}\right)$, or its electromagnetic analogue, gives the Casimir energy in quantum field theory with idealized boundary conditions. (3) Negative powers in $T$ physically represent "divergences" that must be explained or argued away. The term of order $t^{-1}$ is particularly subtle because it corresponds to the first "moment" term in $N$ (also to the topological (index) or Kac term, $O\left(t^{0}\right)$, in $\left.K\right)$. Thus there is no $O\left(\omega^{-1}\right)$ term in $d N / d \omega$, so this term in $T$ has correctly been said to come from low-frequency oscillations of the eigenvalue density rather than high-frequency asymptotics; however, that does not mean that it is one of the nonlocal cylinder-kernel terms. Also, although a divergent local energy density near the boundary does exist, because of an algebraic accident (unrelated to the "moment" issue!) the coefficient of this term in $T(t)$ actually turns out to be zero. However, recent work in physics indicates that the $T$ expansion does not give a trustworthy model of the energy in a realistic system, and in a better model a nonzero $O\left(t^{-1}\right)$ contribution reappears (rendered finite by regularization). The paper works through these observations, roughly in order. 


\section{Mathematical setting and notation}

Although the setting could be greatly generalized, here we assume that $\Omega$ is a compact region in $\mathbb{R}^{d}$ with smooth boundary, and $H$ is an associated positive self-adjoint operator with pure point spectrum. In the situation of most interest for Casimir physics, $-H=\nabla^{2}$ is the Laplacian on scalar functions in $\Omega$ with the Dirichlet boundary condition. We use the two notations

$$
\lambda_{n}=\omega_{n}^{2}
$$

for the $n$th eigenvalue of $H$.

The heat kernel, $K(t, \mathbf{x}, \mathbf{y})$, solves the initial-value problem for $-\frac{\partial u}{\partial t}=H u$ :

$$
u(t, \mathbf{x})=e^{-t H} u(0, \mathbf{x})=\int_{\Omega} K(t, \mathbf{x}, \mathbf{y}) f(\mathbf{y}) d \mathbf{y} .
$$

We have the famous heat kernel expansion,

$$
\begin{aligned}
& K(t, \mathbf{x}, \mathbf{y}) \sim(4 \pi t)^{-d / 2} e^{|\mathbf{x}-\mathbf{y}|^{2} / 4 t} \sum_{s=0}^{\infty} a_{s}(\mathbf{x}, \mathbf{y}) t^{s / 2}, \\
& \operatorname{Tr} K=\int_{\Omega} K(t, \mathbf{x}, \mathbf{x}) d \mathbf{x} \sim(4 \pi t)^{-d / 2} \sum_{s=0}^{\infty} a_{s}[\Omega] t^{s / 2} .
\end{aligned}
$$

Less well known, but more pertinent to vacuum energy, is the cylinder (or Poisson) kernel, $T(t, \mathbf{x}, \mathbf{y})$. It solves the initial-value problem for

$$
\begin{aligned}
& -\frac{\partial^{2} u}{\partial t^{2}}=H u, \quad \lim _{t \rightarrow+\infty} u(t, \mathbf{x})=0: \\
& u(t, \mathbf{x})=e^{-t \sqrt{H}} u(0, \mathbf{x})=\int_{\Omega} T(t, \mathbf{x}, \mathbf{y}) f(\mathbf{y}) d \mathbf{y} .
\end{aligned}
$$

$T$ is the $t$-derivative of another kernel, $\bar{T}$, which solves the same problem as (15) except that $\frac{\partial u(0, \mathbf{x})}{\partial t}$ is the initial data. (Note that $t$ in (2) and (마) can be thought of as related by a Wick rotation to the physical time in nonrelativistic (Schrödinger) and relativistic (wave) equations, respectively.) The cylinder kernel has a trace expansion (33) similar to, and related to, (4), to which we turn in section 6 .

Let $N(\lambda)$ be the number of eigenvalues less than or equal to $\lambda$. Then the density of eigenvalues - the derivative of $N$ - is a distribution, having a Dirac delta function at each eigenvalue. Also, it depends on the variable of integration: $\frac{d N}{d \omega} \neq \frac{d N}{d \lambda}$. Instead,

$$
\frac{d N}{d \omega}=2 \omega \frac{d N}{d\left(\omega^{2}\right)}=2 \sqrt{\lambda} \frac{d N}{d \lambda} .
$$

Famously, the counting function $N$ obeys Weyl's law: As $\omega \rightarrow \infty$,

$$
N\left(\omega^{2}\right) \propto \omega^{d} .
$$

(This holds for a second-order operator in dimension $d$ acting in a compact region $\Omega$.) 


\section{Properties and problems of the Weyl series}

An obvious question is whether (8) is the start of an asymptotic expansion; that is, whether one can write something like

$$
N\left(\omega^{2}\right) \sim \sum_{s=0}^{N} g_{s} \omega^{d-s} .
$$

Because $\operatorname{Tr} K$ is the Laplace transform of $\frac{d N}{d \lambda}$, one can show, by calculations like those in [5], that the coefficients in this series, if it existed, would be determined by the coefficients $a_{s}[\Omega]$ in the rigorous asymptotic series (44). (The Laplace transform of $\lambda^{p-1}$ is proportional to $t^{-p}$, at least for $p>0$.) However, it turns out that only the leading term of the Weyl series is genuinely asymptotic:

$$
N\left(\omega^{2}\right)=\sum_{s=0}^{M} g_{s} \omega^{d-s}+E_{M}(\omega)
$$

where $E_{M}(\omega)$ is usually not of order $O\left(\omega^{d-M-1}\right)$. Instead, when $M>0$, in general $E_{M}$ is of the same order as the previous terms in the series (but is oscillatory). This problem has been understood for ages; the oscillations are related to periodic orbits of the classical system with Hamiltonian $H$.

A related but less well known problem is that the proposed series and its formal derivatives become quite problematical when the exponents of $\omega$ cease to be positive. Consider, for example, the term $g_{d} \omega^{0}$. Its derivative vanishes, so the eigenvalue density $\frac{d N}{d \lambda}$ cannot contain a term proportional to $\lambda^{-1}$. In fact, if a term with that asymptotic behavior did exist, its Laplace transform would contain $\log t$, and the same is true of any negative integral power of $\lambda$; but one knows that such terms do not appear in the heat kernels of second-order differential operators. Where, then, did the heat-kernel coefficient $a_{0}[\Omega]$ come from? It, and the $a_{s}[\Omega]$ with $d-s$ both negative and even, come entirely from the contributions of small values of $\lambda$ to the Laplace transform integral, not from the asymptotic behavior of $N$ at large $\lambda$.

Two approaches have been followed to bring clarity and precision into this seemingly rather muddled situation:

- Riesz-Cesàro means: The heat-kernel coefficients can be related by the Laplace transformation to the coefficients of the asymptotic expansions of sufficiently highorder iterated antiderivatives of the counting function [6, 4].

- Generalized Weyl series and distributional moments: The "missing" coefficients (which "should" accompany negative integral powers of $\lambda$ ) can be identified as those multiplying terms $\delta^{(j)}(\lambda)$ in the asymptotic analysis of $N(\lambda)[7,8]$. These are known as "moment terms" in the theory of distributions [9, 10, 11, 12].

One of our principal goals is to elaborate on the second of these. 


\section{Distributions and moment expansions}

\subsection{Review of general theory}

Recall that distributions are defined as linear functionals on spaces of very well-behaved functions called test functions. A convenient test-function space is

$$
\mathcal{D}\left(\mathbb{R}^{n}\right)=\left\{\phi \in C^{\infty}\left(\mathbb{R}^{n}\right): \phi \text { compactly supported. }\right\}
$$

The corresponding space of distributions is the dual space $\mathcal{D}^{\prime}\left(\mathbb{R}^{n}\right)$, conprising the linear functionals on $\mathcal{D}\left(\mathbb{R}^{n}\right)$ (that are continuous in the weak topology). The action of $f \in \mathcal{D}^{\prime}$ on $\phi \in \mathcal{D}$ is written $\langle f(\mathbf{x}), \phi(\mathbf{x})\rangle$ and generalizes the inner-product integral

$$
\int_{\mathbb{R}^{n}} f(\mathbf{x}) \phi(\mathbf{x}) d \mathbf{x}
$$

to which it reduces when $f$ is a nonsingular ordinary function. These concepts extend to, for example, functions and distributions defined on $\Omega$, with technicalities we shall not discuss here. Also, we sometimes need to refer to distributions taking values in some other vector space (rather than $\mathbb{R}$ or $\mathbb{C}$ ), as in the case of $D^{\prime}(\mathbb{R}, L(\mathcal{X}, \mathcal{H})$ ) in Definition 2 .

Moments are defined as the results of applying distributions to power functions. Because the powers do not have compact support, it is necessary to work with a larger test-function space and hence a more restricted distribution space. Loosely speaking, we need the integral (11) to converge when $\phi(x)=x^{q}$. Let

$$
\mathcal{K}\left(\mathbb{R}^{n}\right)=\left\{\phi \in C^{\infty}\left(\mathbb{R}^{n}\right): \exists q \in \mathbb{N}: D^{\mathbf{k}} \phi(\mathbf{x})=O\left(|\mathbf{x}|^{q-|\mathbf{k}|}\right) \quad \text { as }|\mathbf{x}| \rightarrow \infty\right\} .
$$

(More technically, one defines the space $\mathcal{K}^{[q]}$ of functions satisfying the condition with a fixed $q$, which is a Frechét space under certain seminorms, and then defines $\mathcal{K}$ as the union of those spaces with the inductive limit topology.)

The dual space, $\mathcal{K}^{\prime}\left(\mathbb{R}^{n}\right)$, is the distribution space where moment asymptotic expansions work. Crudely speaking, $f \in \mathcal{K}^{\prime}\left(\mathbb{R}^{1}\right)$ says that $f$ falls off sufficiently fast at infinity that all the moments $\left\langle f(x), x^{q}\right\rangle$ exist [9].

Theorem 1 (moment asymptotic expansion theorem) [12, Sec. 3.3] Function $f$ is in $\mathcal{K}^{\prime}\left(\mathbb{R}^{n}\right)$ if and only if $f(\lambda \mathbf{x})$ admits the moment asymptotic expansion:

$$
f(\lambda \mathbf{x}) \sim \sum_{|\mathbf{k}|=0}^{\infty} \frac{(-1)^{|\mathbf{k}|} \mu_{\mathbf{k}} D^{\mathbf{k}} \delta(\mathbf{x})}{\lambda^{|\mathbf{k}|+n} \mathbf{k} !} \quad \text { as } \lambda \rightarrow \infty .
$$

Here

$$
\mu_{\mathbf{k}}=\left\langle f(\mathbf{x}), \mathbf{x}^{\mathbf{k}}\right\rangle=\left\langle f(\mathbf{x}), x_{1}^{k_{1}} \ldots x_{n}^{k_{n}}\right\rangle, \quad \mathbf{k} \in \mathbb{N}^{n} .
$$

Formula (13) means that if $\phi \in \mathcal{K}\left(\mathbb{R}^{n}\right)$, then for each $M$,

$$
\langle f(\lambda \mathbf{x}), \phi(\mathbf{x})\rangle=\sum_{|\mathbf{k}|=0}^{M}\left\langle\frac{(-1)^{|\mathbf{k}|} \mu_{\mathbf{k}} D^{\mathbf{k}} \delta(\mathbf{x})}{\lambda^{|\mathbf{k}|+n} \mathbf{k} !}, \phi(\mathbf{x})\right\rangle+o\left(\frac{1}{\lambda^{M+n}}\right)
$$

as $\lambda \rightarrow \infty$. Notice that it is an expansion in the parameter $\lambda$, not in the variable $x$ (which will be $\omega$ or $\omega^{2}$ in our spectral applications). It is this change in point of view 
that makes it logically possible to incorporate part of the content of $f$ at small $x$ into the series formally describing the behavior of $f$ at large $x$ [7, 8]. In one dimension, the theorem says that $f \in \mathcal{K}^{\prime}(\mathbb{R})$ is equivalent to the applicability of the moment expansion

$$
f(\lambda \mathbf{x}) \sim \sum_{k=0}^{\infty} \frac{(-1)^{k} \mu_{k} \delta^{(k)}(x)}{\lambda^{k+1} k !} \text { as } \lambda \rightarrow \infty
$$

where $\mu_{k}=\left\langle f(x), x^{m}\right\rangle$.

\subsection{The spectral density}

Let $\mathcal{H}$ be a Hilbert space and let $H$ be a self-adjoint operator with domain $\mathcal{X} \subset \mathcal{H}$. Then $H$ admits a spectral decomposition $\left\{E_{\lambda}\right\}_{\lambda=-\infty}^{\infty}$, such that (in a weak sense)

$$
H=\int_{-\infty}^{\infty} \lambda d E_{\lambda}
$$

In most cases of interest in this paper, this equation is a discrete sum

$$
H=\sum_{n=1}^{\infty} \lambda_{n} P_{n}
$$

where $P_{n}=E_{\lambda_{n}}-E_{\lambda_{n-1}}$ is the orthogonal projection onto the eigenvectors with eigenvalue $\lambda_{n}$.

Definition 2 The spectral density $e_{\lambda}=d E_{\lambda} / d \lambda$ is the distribution in $D^{\prime}(\mathbb{R}, L(\mathcal{X}, \mathcal{H}))$ such that

$$
\left\langle e_{\lambda}, \phi(\lambda)\right\rangle_{\lambda}=\int_{-\infty}^{\infty} \phi(\lambda) d E_{\lambda}
$$

One sometimes denotes $e_{\lambda}$ by $\delta(\lambda-H)$.

For example, the identity operator is $I=\left\langle e_{\lambda}, 1\right\rangle=\int_{-\infty}^{\infty} d E_{\lambda}$, and $H$ itself is $H=\left\langle e_{\lambda}, \lambda\right\rangle=\int_{-\infty}^{\infty} \lambda d E_{\lambda}$, meaning that

$$
(H x \mid y)=\left(\left\langle e_{\lambda}, \lambda\right\rangle_{\lambda} x \mid y\right)=\int_{-\infty}^{\infty} \lambda d\left(E_{\lambda} x \mid y\right) \text { for all } x \in \mathcal{X} \text { and } y \in \mathcal{H}
$$

(Here we write the inner product as $(\cdot \mid \cdot)$ to avoid confusion with the evaluation of a distribution, $\langle\cdot, \cdot\rangle \cdot)$

Let $\mathcal{X}_{n}$ denote the domain of $H^{n}$ and let $\mathcal{X}_{\infty}=\cap_{n=1}^{\infty} \mathcal{X}_{n}$. Then $\left\langle e_{\lambda}, \lambda^{n}\right\rangle=H^{n} \in$ $L\left(\mathcal{X}_{\infty}, \mathcal{H}\right)$ exists. Hence $e_{\lambda} \in \mathcal{K}^{\prime}\left(\mathbb{R}, L\left(\mathcal{X}_{\infty}, \mathcal{H}\right)\right)$

Theorem 3 [12, (6.328)] The moment asymptotic expansion of $e_{\lambda \sigma}$ is

$$
e_{\lambda \sigma} \sim \sum_{k=0}^{\infty} \frac{(-1)^{k} H^{k} \delta^{(k)}(\lambda)}{\sigma^{k+1} k !} \quad \text { as } \sigma \rightarrow \infty
$$

We are now ready to address the Weyl expansion specifically. The trace of $P_{n}$ is the number of eigenvectors with eigenvalue $\lambda_{n}$, so the trace of $E_{\lambda}$ is $N(\lambda)$;

$$
N(x)=\sum_{\lambda_{n} \leq x} 1=\sum_{n} \theta\left(x-\lambda_{n}\right) .
$$


Observe that

$$
N^{\prime}(x)=\sum_{\lambda_{n} \leq x} \delta\left(x-\lambda_{n}\right),
$$

the trace of $e_{\lambda}$. However, if we integrate (16) term by term, we get a wrong answer, inconsistent with (8). The problem is that when we take the trace of $E_{\lambda}$ to get $N(\lambda)$, the result is no longer in $\mathcal{K}^{\prime}$, so the moment expansion theorem does not apply without modification. The (Cesàro-averaged) asymptotic expansion (see [9] and [12, Chap. 6]) of $N(\lambda)$ contains not only the moment terms but also powers of $\lambda$. The exponents may be positive or negative, but negative integers do not occur (being replaced by the moments). Terms of negative half-integer order become literally asymptotic only after repeated indefinite integration to form the Riesz means (see section 7 and [6, 4, 13]).

In the Riesz means of sufficiently high order, the difference between moments and power terms is washed out. Correspondingly, in $\operatorname{Tr} K$ there is no deep distinction between integral and half-integral powers of $t$. Taking the direct product with a onedimensional system (the interval or the one-torus, with $K \propto t^{-\frac{1}{2}}$ ) interchanges odd and even powers of $\sqrt{t}$.

\subsection{Green kernels associated with spectral decompositions}

The same ideas apply to the Green functions, or integral kernels, associated with operators. An operator $H$ on $\mathcal{D}(\Omega)$ can be realized by a distributional kernel $h \in$ $\mathcal{D}^{\prime}(\Omega \times \Omega)$ :

$$
H f(x)=\langle h(x, y), f(y)\rangle_{y} .
$$

For example,

$$
\begin{aligned}
& \langle\delta(x-y), f(y)\rangle=\operatorname{If}(x), \\
& \langle H \delta(x-y), f(y)\rangle=\langle\delta(x-y), H f(y)\rangle=H f(x) .
\end{aligned}
$$

Similarly, $e_{\lambda}$ has an associated kernel $e(x, y ; \lambda) \in \mathcal{K}^{\prime}\left(\mathbb{R}, \mathcal{D}^{\prime}(\Omega \times \Omega)\right)$, such that

$$
\left\langle\langle e(x, y ; \lambda), f(y)\rangle_{y}, \phi(\lambda)\right\rangle_{\lambda}=\left\langle e_{\lambda}, \phi(\lambda)\right\rangle_{\lambda} f(x)=\phi(H) f(x) .
$$

Observe that $\left\langle e(x, y ; \lambda), \lambda^{n}\right\rangle_{\lambda}=H^{n} \delta(x-y)$. So we have

Corollary 4 [11, (61)]

$$
e(x, y ; \sigma \lambda) \sim \sum_{k=0}^{\infty} \frac{(-1)^{k} H^{k} \delta(x-y) \delta^{(k)}(\lambda)}{\sigma^{k+1} k !} \quad \text { as } \sigma \rightarrow \infty .
$$

Let $K(t, x, y)=\left\langle e(x, y ; \lambda), e^{-\lambda t}\right\rangle$ be the heat kernel, as introduced in (2). Then

$$
\begin{aligned}
\left\langle e(x, y ; \lambda), e^{-\lambda t}\right\rangle & =\frac{1}{t}\left\langle e(x, y ; \lambda / t), e^{-\lambda}\right\rangle \\
& \sim \sum_{k=0}^{\infty}\left\langle\frac{(-1)^{k} H^{k} \delta(x-y) \delta^{(k)}(\lambda) t^{k}}{k !}, e^{-\lambda}\right\rangle \\
& =\sum_{k=0}^{\infty} \frac{(-1)^{k} H^{k} \delta(x-y) t^{k}}{k !} \quad \text { as } t \rightarrow 0 .
\end{aligned}
$$


Spectral Invariants and Vacuum Energy

This is a representation of $K$ as a distribution on $\Omega \times \Omega$, so it does not directly give the asymptotic expansion "on diagonal", (41).

\section{Example: The Dirac comb}

Kolomeisky et al. [8] work out some examples where the moment terms can be calculated by the Euler-Maclaurin formula, because the eigenvalues are equally spaced (possibly after a change of variable). Here we study the simplest such case and reproduce the conclusions of [8] by another route.

Theorem 5 [10, Lemma 2.11] If $g \in \mathcal{K}$ and if $\int_{0}^{\infty} g(x) d x$ is defined, then

$$
\begin{aligned}
\sum_{n=1}^{\infty} g(n \varepsilon) & =\left\langle\sum_{n=1}^{\infty} \delta(x-n), g(\varepsilon x)\right\rangle \\
& =\frac{1}{\varepsilon} \int_{0}^{\infty} g(x) d x+\sum_{n=0}^{\infty} \frac{\zeta(-n) g^{(n)}(0)}{n !} \varepsilon^{n}+o\left(\varepsilon^{\infty}\right),
\end{aligned}
$$

where $\zeta(x)$ is the zeta function. Thus $\zeta(-n)$ are the moments in this case. That is,

$$
\sum_{n=1}^{\infty} \delta\left(\frac{x}{\varepsilon}-n\right) \sim \theta(x)+\sum_{n=0}^{\infty} \frac{(-1)^{n} \zeta(-n) \delta^{(n)}(x)}{n !} \varepsilon^{n+1} \quad \text { as } \varepsilon \downarrow 0 .
$$

The generalized Weyl expansion found in [8] is

$$
\sum_{n=1}^{\infty} g\left(\frac{\pi n}{a}\right)=\frac{\pi}{a} \int_{0}^{\infty} g(q) d q-\frac{g(0)}{2}-\frac{\pi g^{\prime}(0)}{12 a}+\frac{\pi^{3} g^{(3)}(0)}{720 a^{3}}-\cdots .
$$

That is, if one defines $G(q)=\sum \delta(q-n)$, then

$$
G\left(\frac{a q}{\pi}\right) \sim \frac{a}{\pi}-\frac{\delta(q)}{2}+\frac{\pi \delta^{\prime}(q)}{12 a}-\frac{\pi^{3} \delta^{(3)}(q)}{720 a^{3}}+\cdots \quad \text { as } a \rightarrow \infty .
$$

Here $a$ denotes the radius of the one-dimensional interval, half the distance between the (Dirichlet) boundaries.

By Theorem 5, if $g \in \mathcal{K}$ and if $\int_{0}^{\infty} g(x) d x$ is defined, then

$$
\sum_{n=1}^{\infty} g(n \varepsilon) \sim \frac{1}{\varepsilon} \int_{0}^{\infty} g(x) d x+\sum_{n=0}^{\infty} \frac{\zeta(-n) g^{(n)}(0)}{n !} \varepsilon^{n} \quad \text { as } \varepsilon \downarrow 0 .
$$

If we let $\varepsilon=\frac{\pi}{a}$ and compute the zeta-function terms, we obtain the same equation as (24). Note that the first term is a Weyl (high-frequency asymptotic) term and the rest are moment terms, related to the low-frequency content of $N^{\prime}$.

The most elementary application of this expansion is when the eigenvalues are proportional to $n^{2}$. Then

$$
N(x)=\sum_{n \geq 0} \theta\left(x-n^{2}\right), \quad N^{\prime}(x)=\sum_{n \geq 0} \delta\left(x-n^{2}\right) .
$$


If we set $g\left(x^{2}\right)=f(x)$, we can then calculate

$$
\begin{aligned}
\left\langle\sum_{n=1}^{\infty} \delta\left(x-n^{2}\right), g(\varepsilon x)\right\rangle & =\sum_{n=1}^{\infty} f\left(\varepsilon^{1 / 2} n\right) \\
& \sim \frac{1}{2 \varepsilon^{1 / 2}} \int_{0}^{\infty} \frac{g(x)}{\sqrt{x}} d x \quad \text { as } \varepsilon \downarrow 0
\end{aligned}
$$

for the first term. But, actually, the first term is all: For the moment terms we can calculate the moments of $N^{\prime}$ to be

$$
\mu_{k}=\left\langle\sum_{n \geq 0} \delta\left(x-n^{2}\right), x^{k}\right\rangle=\zeta(-2 k)=0 .
$$

That is,

$$
\sum_{n=1}^{\infty} \delta\left(\frac{x}{\varepsilon}-n^{2}\right)=\frac{\theta(x)}{2 \varepsilon^{1 / 2} \sqrt{x}}+o\left(\varepsilon^{\infty}\right) \quad \text { as } \varepsilon \downarrow 0 .
$$

As an example of the example, let $H y=y^{\prime \prime}$ be considered on the domain $\mathcal{X}=\left\{y \in C^{2}[0, \pi]: y(0)=y(\pi)=0\right\}$ inside $L^{2}[0, \pi]$. The eigenvalues are $\lambda_{n}=n^{2}$, $n=1,2,3, \ldots$, with normalized eigenfunctions $\phi_{n}(x)=\sqrt{2 / \pi} \sin n x$. Therefore,

$$
e(x, y ; \lambda)=\frac{2}{\pi} \sum_{n=1}^{\infty} \sin n x \sin n y \delta\left(\lambda-n^{2}\right),
$$

and

$$
\begin{aligned}
e(x, x, \lambda) & =\frac{2}{\pi} \sum_{n=1}^{\infty} \sin ^{2} n x \delta\left(\lambda-n^{2}\right) \\
& =\frac{1}{\pi} \sum_{n=1}^{\infty}(1-\cos 2 n x) \delta\left(\lambda-n^{2}\right) .
\end{aligned}
$$

Hence

$$
e(x, x, \lambda / t) \sim \frac{1}{\pi} \frac{\theta(\lambda) t^{1 / 2}}{2 \sqrt{\lambda}} \quad \text { as } t \downarrow 0
$$

for $0<x<\pi$. From this we recover the asymptotics of the heat kernel:

$$
\begin{aligned}
\left\langle e(x, x ; \lambda), e^{-\lambda t}\right\rangle & \sim \frac{1}{2 \pi t^{1 / 2}} \int_{0}^{\infty} \frac{e^{-\lambda}}{\sqrt{\lambda}} d \lambda \\
& =\frac{1}{(4 \pi t)^{1 / 2}} \quad \text { as } t \downarrow 0 .
\end{aligned}
$$

This result is nonuniform in $x$, so it does not give the correct trace expansion (4), which contains an additional term representing the effect of the Dirichlet (or alternative) boundaries.

Furthermore, we can study the relation to the expansion of the cylinder kernel. We know from (28) that

$$
\sum_{n=1}^{\infty} \delta\left(\frac{x}{\varepsilon}-n^{2}\right)=\frac{\theta(x)}{2 \varepsilon^{1 / 2} \sqrt{x}}+o\left(\varepsilon^{\infty}\right) \quad \text { as } \varepsilon \downarrow 0 .
$$


If we set $x=\omega^{2}$, we might expect

$$
\sum_{n=1}^{\infty} \delta\left(\frac{\omega^{2}}{\varepsilon}-n^{2}\right)=\frac{\theta(\omega)}{2 \varepsilon^{1 / 2} \omega}+o\left(\varepsilon^{\infty}\right) \quad \text { as } \varepsilon \downarrow 0 .
$$

But in fact, it's not so trivial:

$$
\sum_{n=1}^{\infty} \delta\left(\omega^{2}-n^{2}\right)=\sum_{n=1}^{\infty} \frac{1}{2 n}[\delta(\omega-n)+\delta(\omega+n)],
$$

so by (23) we have

$$
\begin{aligned}
\sum_{n=1}^{\infty} \delta\left(\frac{\omega^{2}}{\varepsilon}-n^{2}\right) & \sim \frac{\theta(\omega)}{2 \varepsilon^{1 / 2} \omega}+\sum_{n=0}^{\infty} \frac{(-1)^{n+1} \zeta(-n) \delta^{(n+1)}(\omega)}{2(n+1) !} \varepsilon^{\frac{n+1}{2}} \\
+o\left(\varepsilon^{\infty}\right) & \text { as } \varepsilon \downarrow 0 .
\end{aligned}
$$

The significance of the extra terms in (32) will become clear in the next two sections.

\section{The cylinder kernel}

Because the material of this section and the next has been extensively covered before [6, 4, 13], we shall be relatively brief. But to atone for a certain vagueness at certain points in earlier sections, we give complete and precise formulas. Please consult those papers for references to the fundamental work of Hardy and Hörmander on which the theorems are based.

The cylinder kernel of (6) has the trace expansion

$$
\sum_{n=1}^{\infty} e^{-t \omega_{n}}=\operatorname{Tr} T=\sum_{s=0}^{\infty} e_{s} t^{-d+s}+\sum_{\substack{s=d+1 \\ s-d \text { odd }}}^{\infty} f_{s} t^{-d+s} \log t
$$

It is convenient to redefine the expansion coefficients in the heat trace (4) by

$$
\sum_{n-1}^{\infty} e^{-t \omega_{n}^{2}}=\operatorname{Tr} K=\sum_{s=0}^{\infty} b_{s} t^{(-d+s) / 2}
$$

when treating its relations with the cylinder trace and with Riesz means of $N$.

Theorem 6 The coefficients in the cylinder and heat expansions are related by

$$
e_{s}=\pi^{-1 / 2} 2^{d-s} \Gamma\left(\frac{d-s+1}{2}\right) b_{s} \quad \text { if } d-s \text { is even or positive, }
$$

whereas if $d-s$ is odd and negative,

$$
f_{s}=\frac{(-1)^{(s-d+1) / 2} 2^{d-s+1}}{\sqrt{\pi} \Gamma((s-d+1) / 2)} b_{s}, \quad \text { but } e_{s} \text { is undetermined by the } b_{r} .
$$

The new coefficients $e_{s}$ (with $d-s$ odd and negative) are new spectral invariants. They are nonlocal in their dependence on the geometry of $\Omega$. The first one, $e_{d+1}$, has the interpretation of renormalized Casimir vacuum energy in quantum field theory. 


\section{Riesz means, old and new}

Riesz means are a generic tool of long standing, but we consider only those of the counting function, $N$. The "old" Riesz means (with respect to $\lambda$ ) are defined by the $\alpha$-fold iterated (simplex) indefinite integration:

$$
R_{\lambda}^{\alpha} N(\lambda)=\frac{1}{\alpha !} \lambda^{-\alpha} \int^{\lambda} \cdots \int N(\tilde{\lambda}) d \tilde{\lambda} .
$$

But we may also have Riesz means with respect to $\omega=\sqrt{\lambda}$ :

$$
R_{\omega}^{\alpha} N(\omega)=\frac{1}{\alpha !} \omega^{-\alpha} \int^{\omega} \cdots \int N\left(\tilde{\omega}^{2}\right) d \tilde{\omega} .
$$

Theorem 7 There exist asymptotic formulas of the forms

$$
\begin{aligned}
& R_{\lambda}^{\alpha} N=\int_{s=0}^{\alpha} a_{\alpha s} \lambda^{(d-s) / 2}+O\left(\lambda^{(d-\alpha-1) / 2}\right), \\
& R_{\omega}^{\alpha} N=\int_{s=0}^{\alpha} c_{\alpha s} \omega^{d-s}+\sum_{\substack{s=d+1 \\
s-d \text { odd }}}^{\alpha} d_{\alpha s} \omega^{d-s} \log \omega+O\left(\omega^{d-\alpha-1} \log \omega\right) .
\end{aligned}
$$

Contrast (9). In words, the oscillations that prevent the Weyl series from being asymptotic beyond the first term are averaged out by the integrations, so that the corresponding series for a Riesz mean is a valid asymptotic approximation to a certain higher order.

Only the coefficients $a_{s s}$ are truly important; the $a_{\alpha s}$ with $s<\alpha$ contain redundant information.

Theorem 8 The heat-kernel coefficients are proportional to the old Riesz means:

$$
b_{s}=\frac{\Gamma((d+s) / 2+1)}{\Gamma(s+1)} a_{s s} .
$$

The cylinder-kernel coefficients are related to the new Riesz means by

$$
\begin{aligned}
e_{s} & =\frac{\Gamma(d+1)}{\Gamma(s+1)} c_{s s} \quad \text { if } d-s \text { is even or positive, } \\
f_{s} & =-\frac{\Gamma(d+1)}{\Gamma(s+1)} d_{s s}, \quad e_{s}=\frac{\Gamma(d+1)}{\Gamma(s+1)}\left[e_{s s}+\psi(d+1) d_{s s}\right]
\end{aligned}
$$

if $d-s$ is odd and negative.

It is therefore no surprise that the asymptotic coefficients of the old and new Riesz means are related by formulas [6] very similar to those relating the heat and cylinder coefficients. In particular, when $d-s$ is odd and negative, $c_{s s}$ is undetermined by the $a_{r r}$. There are integral operations leading from old Riesz means to new Riesz means and vice versa:

- When going old $\rightarrow$ new, the new $c_{s s}$ arise from the lower limit of integration, bringing in new information about $N\left(\omega^{2}\right)$ at low frequencies.

- When going new $\rightarrow$ old, the $c_{s s}$ are multiplied by numerical coefficients that turn out to equal 0 when $d-s$ is odd and negative, so their information is lost in those cases. 


\section{One very special term}

The term of order $t^{0}$ in a heat kernel is geometrically dimensionless and has topological significance. It counts eigenfunctions with eigenvalue zero.

More precisely, when $H_{1}=A^{*} A$ and $H_{2}=A A^{*}$,

$$
\operatorname{Tr} K\left[H_{1}\right]-\operatorname{Tr} K\left[H_{2}\right]
$$

is independent of $t$ (only the $O\left(t^{0}\right)$ terms fail to cancel) and equals the index of the operator A. Kolomeisky et al. [8] call it the Kac term. We propose that index term is more descriptive.

The index term in $K$ corresponds to a constant term in (the averaged) $N$. More precisely, since $N(\lambda)=0$ for $\lambda<0$, the term is a multiple of the Heaviside function, $\theta(\lambda)$. Therefore, it gives rise to a multiple of the Dirac delta distribution in the eigenvalue density $d N / d \lambda$ or $d N / d \omega$. Thus, whether this term is a moment or a high-frequency asymptotic term depends on which function one is looking at.

Because there is no $\log \omega$ term in $N$, there is no $O\left(\omega^{-1}\right)$ term in $d N / d \omega$. Therefore, it is correctly said that the index term comes from the low-frequency, not high-frequency, behavior of the spectral density. Note, however, that it is a true heat-kernel term, locally determined by the geometry, not one of the new cylinder-kernel terms.

The index term corresponds to a term $O\left(t^{0}\right)$ in the cylinder trace, $T$. There is no $O(\log t)$ term. Hence there is no $O\left(t^{-1}\right)$ term in $d T / d t$. $d T / d t$ has the physical interpretation of ( -2 times) the vacuum energy of a scalar field, subjected to timelike point-splitting regularization (see references in [14, 15]). Negative powers of $t$ here represent buildups of energy against the idealized boundary, which must be absorbed into the description of the boundary itself (renormalization).

We see that no renormalization is needed in order $t^{-1}$; nevertheless, calculations of local energy density show that large boundary energy is indeed there! (Energy density proportional to $z^{-2}(z=$ distance to boundary) formally implies energy proportional to $t^{-1}$.)

Candelas [16] correctly stresses the physical necessity of the $O\left(z^{-2}\right)$ energy density and argues (apparently incorrectly) that an $O\left(t^{-1}\right)$ term in the total energy arises from $d N / d \omega$. Other authors [17, 8] correctly point out that no such term exists. Here we attempt to dispose of this controversy.

First, in timelike point-splitting regularization the large energy density near the boundary is compensated by a larger opposite-sign density concentrated even closer to the boundary. Therefore, there is no mathematical contradiction between the local and global statements.

Second, there are now physical reasons to believe that for certain purposes, spacelike point-splitting gives a more trustworthy model of the energy in a realistic system [14, 15]. In that framework the contribution of the index term does not vanish after all.

Third, quite apart from the technical criticisms of [16] in [8], a close examination of [16] shows that its argument (as concerns the total energy, not the boundary energy 
density) does not note the possibility that the overall coefficient of the term in question might turn out to be zero. In fact, as just remarked, it is zero in timelike but not in spacelike point-splitting.

Thus a 30-year-old controversy has been revived, resolved, and rendered irrelevant in roughly the same year (2012-13).

In summary, the index term has a number of special properties, which are not particularly closely related to each other:

- It does not contribute to the total energy in timelike regularization, because its contribution to $\operatorname{Tr} T$ is killed by the differentiation.

- Because it sits on the boundary between moments and high-frequency asymptotics, there is no corresponding $O\left(\lambda^{-1}\right)$ term in the eigenvalue density.

- Some moments (low-frequency contributions) in $\operatorname{Tr} T$ are new spectral invariants that do not appear in $\operatorname{Tr} K$. However, this term is not one of them. They start immediately afterwards.

There is a tendency for these three issues to become muddled together in our thinking.

\section{Summary of the subtleties}

- Certain powers in the heat-kernel expansion match moment (delta function) terms (not powers) in the "generalized Weyl expansion". A generalized Weyl expansion that is introduced in the paper [8] can be realized as the moment asymptotic expansion of the Dirac comb $\sum_{n=1}^{\infty} \delta(x / \varepsilon-n)$ acting on a test function.

- The missing powers match terms in the cylinder-kernel expansion. These new, nonlocal spectral invariants show up in the Riesz-Cesàro asymptotics of $N$ with respect to $\omega$ (but not $\left.\omega^{2}\right)$.

- Some confusion and controversy in the physics literature is related to this fact: The term in the $t$-derivative of the cylinder kernel trace corresponding to the "index" term in the heat kernel trace vanishes because of an algebraic accident, but nevertheless quantum field theory predicts a divergent boundary energy density proportional to that spectral invariant. That this term is a moment, not a highfrequency part of the eigenvalue density, is beside the point. Recent physics suggests that this method of regularizing the energy is not reliable anyway.

\section{Acknowledgments}

This research was supported by National Science Foundation Grants PHY-0968269 and PHY-0968448. Y. Yang thanks the Texas A\&M Mathematics Department for hospitality while much of the work was done. Its dissemination was aided by invitations to participate in the Special Session on Topics in Spectral Geometry and Global Analysis, AMS Western Section Meeting, Albuquerque, and in the Casimir Physics Session, École 
de Physique, Les Houches. We both thank our mentor in distribution theory, Ricardo Estrada.

\section{References}

[1] Balian R and Bloch C 1971 Asymptotic evaluation of the Green's function for large quantum numbers Ann. Phys. (NY) 63 592-606

[2] Brack M and Bhaduri R K 1997 Semiclassical Physics (Reading:Addison-Wesley)

[3] Christensen S M 1976 Vacuum expectation value of the stress tensor in an arbitrary curved background: The covariant point-separation method Phys. Rev. D 14 2490-2501

[4] Fulling S A 2003 Systematics of the relationship between vacuum energy calculations and heatkernel coefficients J. Phys. A 36 6857-6873

[5] Fulling S A 1982 The local geometric asymptotics of continuum eigenfunction expansions I SIAM J. Math. Anal. 13 891-912

[6] Fulling S A and Gustafson R A 1999 Some properties of Riesz means and spectral expansions Electron. J. Diff. Eqs. 19996

[7] Dowker J S 2002 1. The counting function. 2. Hybrid boundary conditions Nucl. Phys. B (Proc. Suppl.) 104 153-156

[8] Kolomeisky E B, Zaidi H, Langsjoen L, and Straley J P 2013 Weyl problem and Casimir effects in spherical shell geometry Phys. Rev. A 87042519

[9] Estrada R 1998 The Cesàro behaviour of distributions Proc. Roy. Soc. Lond. A 454 2425-2443

[10] Estrada R, Gracia-Bondía J M, and Várilly J C 1998 On summability of distributions and spectral geometry Commun. Math. Phys. 191 219-248

[11] Estrada R and Fulling S A 1999 Distributional asymptotic expansion of spectral functions and of the associated Green kernels Electron. J. Diff. Eqs. 19997

[12] Estrada R and Kanwal R P 2002 A Distributional Approach to Asymptotics: Theory and Applications (Boston:Birkhäuser)

[13] Fulling S A 2004 Global and local vacuum energy and closed orbit theory Quantum Field Theory under the Influence of External Conditions, ed K A Milton (Princeton:Rinton) pp 166-174

[14] Estrada R, Fulling S A, and Mera F D 2012 Surface vacuum energy in cutoff models: Pressure anomaly and distributional gravitational limit J. Phys. A 45455402

[15] Milton K A, Kheirandish F, Parashar P, Abalo P K, Fulling S A, Bouas J D, and Carter H 2013 Investigations of the torque anomaly in an annular sector. I. Global calculations, scalar case Phys Rev D 88025029

[16] Candelas P 1982 Vacuum polarization in the presence of dielectric and conducting surfaces Ann. Phys. (NY) 143 241-295

[17] Bernasconi F, Graf G M, and Hasler D 2003 The heat kernel expansion for the electromagnetic field in a cavity Ann. H. Poincaré 4 1001-1013 